\title{
A Note on the "Optimal" Design of Disc-Shaped Heat Exchangers ${ }^{\dagger}$
}

\section{Enrico Sciubba}

Department of Mechanical and Aerospace Engineering, University of Roma 1 "La Sapienza", Via Eudossiana 18, 00184 Roma, Italy; enrico.sciubba@uniroma1.it

† Presented at the First World Energies Forum, 14 September-05 October 2020; Available online: https://wef.sciforum.net/.

Published: 12 September 2020

\begin{abstract}
The continuous quest for improving the performance of heat exchangers, together with evermore stringent volume and weight constraints, especially in enclosed applications (engines, electronic devices), stimulates the search for compact, high-performance units. One of the shapes that emerged from a vast body of research is the disc-shaped heat exchanger, in which the fluid to be heated/cooled flows through radial, often bifurcated, channels inside of a metallic disc. The disc, in turn, exchanges heat with the heat/cold source (the environment or another body). Several studies have been devoted to the identification of an "optimal shape" of the channels: Most of them are based on prime principles, though numerical simulations abound as well. The present paper demonstrates that, for all engineering purposes, there is only one correct design procedure for such a heat exchanger, and that this procedure depends solely on the technical specifications (exchanged thermal power, materials, surface quality): The design, in fact, reduces to a zero-degree of freedom problem! The argument is described in detail, and it is shown that a proper application of the constraints completely identifies the shape, size and similarity indices of both the disc and the internal channels. The goal of this study is not that of "inventing" a novel heat exchanger design procedure, but that of demonstrating that -in this as in many similar cases- a straightforward application of prime principles and of diligent engineering rules may generate "optimal" designs. Of course, the resulting configurations may be a posteriori tested as to their performance, their irreversibility rates, their compliance with one or the other "techno-economical optimization methods", but it is important to realize that they enjoy a sort of "embedded" optimality.
\end{abstract}

Keywords: bifurcated flows; disc-shaped heat exchangers; fluid transport; heat transfer

\section{Introduction}

Consider the general structure of a Disc-Shaped Heat Exchanger element, DSHE (Figure 1). It consists of a disc of radius Rext (in the following referred to as " $\mathrm{R}_{\mathrm{b}}$ ") internally cooled (or heated: In the remaining of this paper, for the sake of simplicity, we shall refer only to internal cooling) by a series of circular channels that originate from an axial inlet A and develop radially outwards in a branching fashion, so that the cooling fluid is collected by a toroidal manifold placed on the external periphery of the disc. The channels may have different diameters and lengths. 

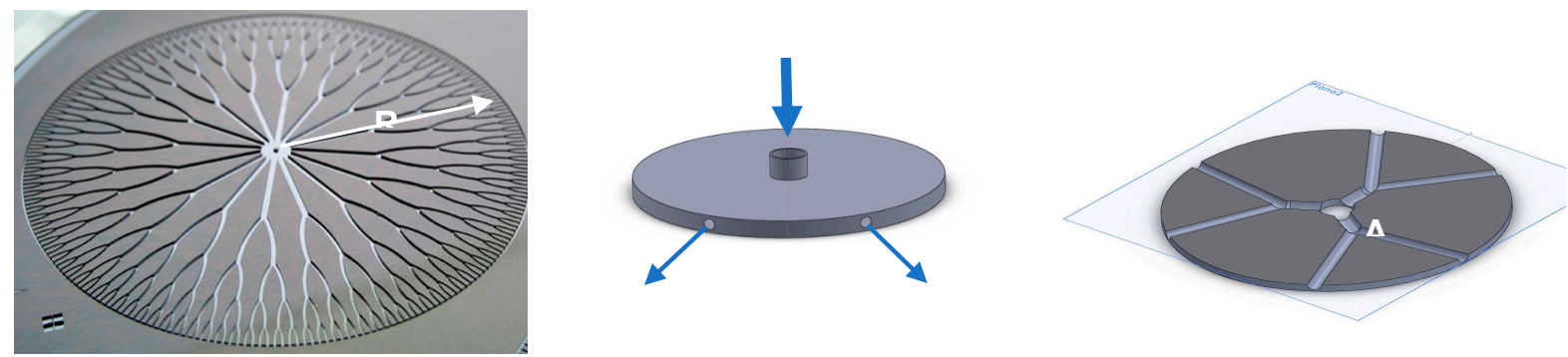

Figure 1. Sketch of a Disc-Shaped Heat Exchanger (DSHE).

One of the first problems designers must solve is the identification of the, possibly unique, set of lengths $L_{j}$ and diameters $d_{j}$ of the channels. Another design issue is to determine whether a larger number of branchings (i.e., a "more dendritic" structure) leads to a performance improvement, and how to quantify the correlation between the number of branches and the DSHE performance. Finally, it must be investigated whether the branching angles $\beta_{j}$ have an influence on the overall heat exchange characteristics of the device.

Historically, the problem has been approached by reformulating it in terms of "heat gain" of the cooling fluid versus "pressure drop" along the channels. A somewhat more sophisticated approach is that of calculating both the thermal entropy generation rate (due to the local temperature gradients in the fluid and in the disc) and its viscous counterpart (due to friction) and minimizing their sum: Since the former is proportional to the HE efficiency and the latter to the pumping power, a sort of global optimum is reached that balances benefits and costs of the energy exchange. More recently, approaches have been proposed that introduce material costs considerations, either by applying Thermo-Economic principles or Exergy Analysis.

All of these studies have identified and used one or more similarity parameters that can be directly linked to performance indicators: These "shape parameters" are the ratio of the diameters of successive branches, $\delta_{j}=d_{j+1} / d_{j}$; the ratio of successive lengths, $\lambda_{j}=L_{j+1} / L_{j}$; the ratio of the diameter of the first branch to the disc radius, $\delta_{0}=d_{0} / R_{z b}$. Quite obviously, the more branches are etched inside of the disc, the more uniform the heat extraction from the solid material, and the better the performance: Thus, both the initial number of "sectors" in which the disc is subdivided (first branches, $z_{0}$ ) and the total number of branchings, $z_{b}$, have an influence on the performance of the device.

Investigators have taken different approaches to the solution: In general, global design optima have been identified by means of more or less "allometric" formulae (An allometric correlation is one in which the scaling relationship between a relevant attribute and a characteristic length of the problem depends on some power of the characteristic length itself. For example, in biology, it is established opinion that the metabolic rate depends on the "body size" (i.e., on the cube of some characteristic body length)) that define $\delta_{j}$ the and/or the $\lambda_{j}$ for a given $\delta_{0}$. Only a few studies investigate the influence of the number of branchings. The few numerical studies available are usually based on a pre-assigned initial choice of $z_{0}$ and take advantage of the circumferential symmetry of the problem to simulate only a sector spanning $2 \pi /$ zo radians (Figure 2 ). 


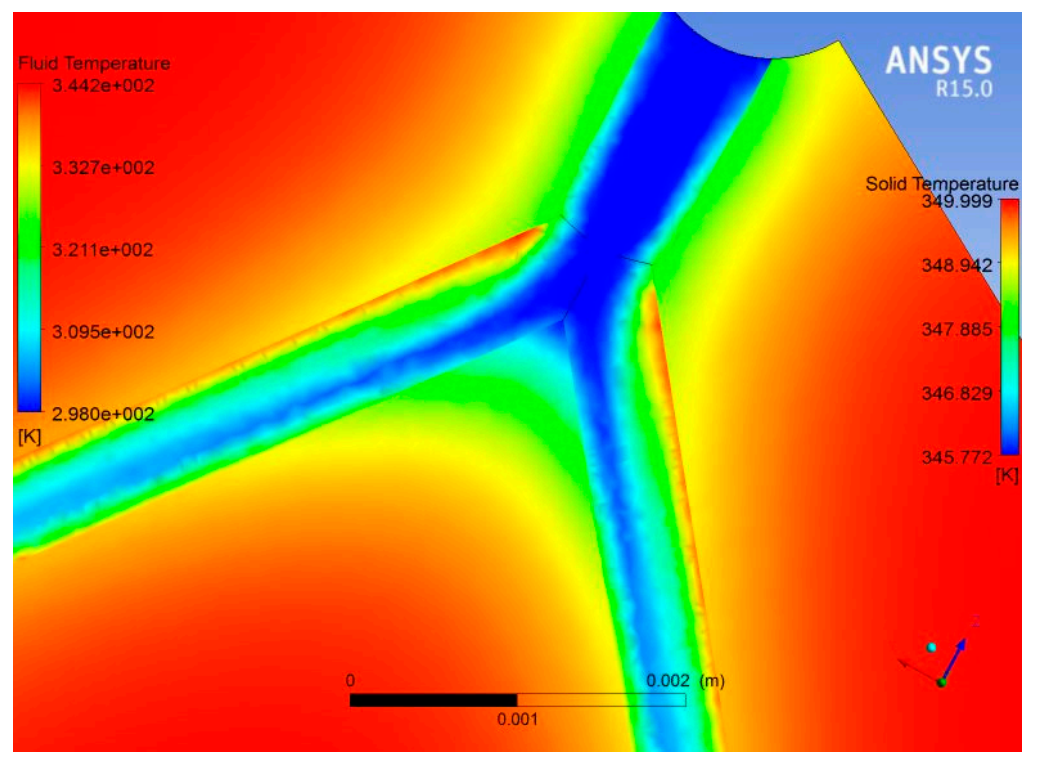

Figure 2. "Typical" numerical simulation (CFD) of a DSHE [1].

The most popular and comprehensive theoretical model is Bejan's Constructal Theory (CT in the following), based on a clever generalization of an "optimal ratio" derived over a century ago (1903-1917) by the Swiss physiologist (and future Nobel laureate) Walter Rudolf Hess [2,3] for a model of blood flow in arterioles. The same result was "rediscovered" by the American physiologist Cecil Dunmore Murray in 1926 [4-6], and the correlation is since referred to as the "Hess-Murray law". The method was innovative for the times: Under the assumption that blood or lymph circulation in living organisms is governed by a "work minimization" principle, Hess and Murray proved that, under certain conditions, there exists an "optimal branching ratio" of $\delta=\frac{\mathrm{d}_{i+1}}{\mathrm{~d}_{i}}=\frac{1}{\sqrt[3]{2}}=$ 0.7937 between the diameters of two successive branchings. In spite of the poor agreement between the dictates of the $\mathrm{H}-\mathrm{M}$ law and both experimental and numerical results (The $\mathrm{H}-\mathrm{M}$ law underwent extensive theoretical and experimental reassessment in the second half of the 20th century, and the results indicate that the law is sufficiently accurate for the smallest vessels ( $d$ of the order of a millimeter) but fails for the larger ones (like arteries) [7-11]. Moreover, it cannot be successfully extended to turbulent flows. Recent comparisons with numerical investigations of branched flows led to similar conclusions. See [12] for a review.), this "cubic root of 2" correlation was successfully reformulated and extended, resulting in "a general theory of material and energy transport" [13-17], Bejan's Constructal Theory ("CT" in the following). In spite of its great success [18-22], CT does not really provide a satisfactory answer to the problem of choosing the initial number of sectors in which the disc must be divided $(z 0)$, the total number of branchings $(z b)$ and has shown no clear advantage in terms of the "optimality" of its design (for instance, it has been demonstrated that CT works well only as long the flow remains laminar [23,24]. Furthermore, it does not address several practical design points, like the channels wall roughness, the viscous losses at each split, etc. Moreover, as will be shown in the following, neither produces DSHE "optimal geometries".) [7-10,25,26]. Some of the latest works in CT actually seem to disregard some phenomenological sides of the problems $[18,27,28]$.

This paper presents a description and a detailed discussion of a completely feasible, realistic, and rational engineering procedure for the design of a disc-shaped HE. The procedure rests on the rigorous application of thermo-fluid dynamic principles and on a careful analysis of the implications of the selected topology $\left(z_{0}, z b\right)$ on the global device performance. Section 2 presents a topological description of the disc, Section 3 demonstrates that a physically correct design procedure leads to an under-specified problem (more variables than equations), and Section 4 shows how the imposition of a correct set of constraints can make the problem well-posed. Section 5 provides two examples of the advantages of a practical application of the procedure. 


\section{The Physiology of a Disc Heat Exchanger as a Function of Its Operational Mode}

As stated above, the relevant topological parameters of DSHE are:

i - the ratio of the diameters of successive branches, $\delta_{j}=d_{j+1} / d_{j}$;

ii - the ratio of successive lengths, $\lambda_{j}=L_{j+1} / L_{j}$;

iii - the shape ratio defined as the ratio of the diameter of the first branch to the disc radius, $\delta_{0}=d_{0} / R$;

iv - the initial number of "sectors" in which the disc is subdivided, $z$;

$\mathrm{v}$ - the total number of branchings, $z b$.

The above parameters define the shape of the DSHE: Its size depends obviously on the design specifications. There are several possible applications for a DSHE, but they can be reduced to a single fundamental type of operation. For the sake of simplicity, we shall consider here the case in which the fluid flowing in the channels cools the disc (i.e., from the point of view of the fluid, the DSHE is a heater). As shown in Figure 3, the cooling fluid flows through the disc's internal channels, and the hot source is either the surroundings (via convection on the DSHE external surfaces, Figure 3a), surface heating by conduction or electrical (Figure 3b), or surface heating on one side and convection cooling on the other side (Figure 3c). In all three configurations, the coolant absorbs heat from the bulk material of the disc, which, in turn, receives a continuous heat influx on one or both of its surfaces.

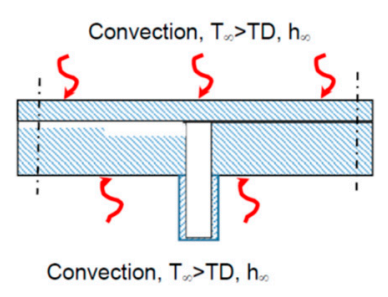

(a) Convection both sides

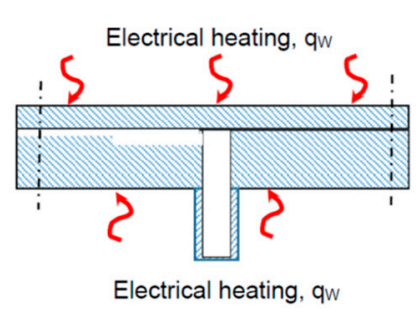

(b) Conduction both sides

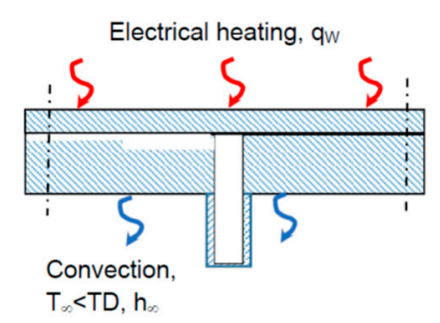

(c) Conduction and convection

Figure 3. Fundamental modes of operation of a DSHE (here, as a heater).

For a DSHE-heater, a suitable set of specifications includes:

(a) The thermal flux $q_{\text {in }}$ the DSHE receives by conduction or electrical input -if any;

(b) The inlet and outlet coolant temperatures $T_{\text {in }}$ and $T_{\text {out }}$;

(c) The final $\Delta T$ between the disc and the fluid: $T_{D}-T_{\text {out }}$;

(d) The average temperature $T_{\text {ext }}$ of the immediate surroundings;

(e) The density $\rho_{f}$, specific heat $c_{p, f}$, viscosity $\mu_{f}$ of the coolant;

(f) The density $\rho_{D}$ and specific heat $c_{p, D}$ of the disc material;

(g) An average heat transfer coefficient for the convection on the outside surface of the disc, hext if present.

The net heat input $Q_{\text {des }}$ depends on the type of operation, and the respective formulae are provided in Table 1 . Once the net heat input $Q_{\text {des }}$ is known, the coolant mass flow rate can be calculated from the global energy balance

$$
m_{\text {in }}=\frac{Q_{\text {des }}}{\eta_{D S H E} c_{p}\left(T_{\text {out }}-T_{\text {in }}\right)}
$$

where $\eta_{D S H E}$ is as yet unknown, and must be assumed as a first trial value (it can be refined at a later design stage, or estimated on the basis of semi-empirical charts). Since the numerical value of $\eta_{D S H E}$ is inessential for the design procedure outlined here, a $90 \%$ efficiency will be assumed in the remaining of the calculations (The DSHE efficiency depends in fact mainly on the ratio s/R: The higher this ratio, the higher the convection losses on the disc peripheral surface. This dependence is not considered in this study.). 
Table 1. Definition of the design DSHE thermal load.

\begin{tabular}{|c|c|c|}
\hline Type of Operation (Heater) & $\begin{array}{l}\text { Needed Design } \\
\text { Specifications }\end{array}$ & $Q_{\text {des }}$ \\
\hline Heat input by convection on both sides & $h_{\text {ext, }} \mathrm{T}_{\mathrm{ext}}, \mathrm{T}_{\mathrm{D}}$ & $Q_{\text {des }}=2 \pi h_{\text {ext }} R^{2} \Delta T_{D}$ \\
\hline Electrical or conduction heating on both sides & $\mathrm{q}_{\mathrm{in}, \mathrm{SD}}, \mathrm{kD}_{\mathrm{D}}$ & $Q_{\text {des }}=2 \pi q_{i n} R^{2}$ \\
\hline $\begin{array}{l}\text { Electrical or conduction heating on one surface, } \\
\text { cooling by free convection on the opposite one }\end{array}$ & $h_{\text {ext }}, \mathrm{T}_{\text {ext }}, \mathrm{T}_{\mathrm{D}}, \mathrm{q}_{\mathrm{in}}, \mathrm{SD}, \mathrm{k}_{\mathrm{D}}$ & $\begin{array}{l}Q_{\text {des }} \\
=2 \pi R^{2}\left(q_{\text {in }}\right. \\
\left.-h_{\text {ext }} \Delta T_{D}\right)\end{array}$ \\
\hline
\end{tabular}

From Equation (1) and Table 1 we see that the required mass flow rate of coolant is proportional to the square of disc radius $\mathrm{R}$. If the former is specified by process requirements, then $\mathrm{R}$ has a unique value (Equation (2a)). Alternatively, if $R$ is dictated by space requirements, the mass flow rate of coolant is given by Equation $(2 b)$ :

$$
\begin{gathered}
R=\left(\frac{\eta_{D S H E} m_{f} c_{p}\left(T_{\text {out }}-T_{\text {in }}\right)}{F_{D S H E}}\right)^{0.5} \\
m_{f}=\frac{F_{D S H E}}{\eta_{D S H E} c_{p}\left(T_{\text {out }}-T_{\text {in }}\right)} \mathrm{R}^{2}
\end{gathered}
$$

with $F_{D S H E}=2 \pi h_{\text {ext }} \Delta T_{D}, 2 \pi q_{\text {in }}$ or $2 \pi\left(q_{\text {in }}-h_{\text {ext }} \Delta T_{D}\right)$.

Notice that when the above formulae are used for cases (a) and (c) their results are approximate because the disc is likely to have a radially variable surface temperature, meaning that $\Delta T_{D}$ is not a constant but a function of $r$ : If more accuracy is required, the value of $\mathrm{R}$ can be calculated iteratively using the final results of the procedure specified below.

A designer would, in general, select the range of Reynolds number for the inlet flow. This choice is guided by two fundamental knowledge bits in heat transfer: The Nusselt number in internal channels grows approximately with the $\operatorname{Re}^{1 / 3}$, while the pressure drop in the channels grows with $\operatorname{Re}^{2}$. Therefore, it is advisable to select $\mathrm{Re}$ in the vicinity of the transition (where the friction factor is lowest) but as high as possible (to increase $\mathrm{Nu}$ ). Once $R e_{\text {in }}$ is specified:

$$
d_{i n}=\frac{4 m_{i n}}{\pi \mu R e_{i n}}
$$

The above equations provide the designer with a tentative size $R$ of the disc, the diameter $d_{\text {in }}$ of the inlet tube, and the required coolant mass flow rate. On this basis, an "intrinsically optimal" (the claim for optimality is set within quotes because no optimization proper is performed when applying the proposed design procedure. Only fluid-thermodynamic constraints are imposed (the constancy of Re and the Graetz assumption, see below), and the optimal solution arises simply out of symmetry and of the geometric features of the branchings.) design procedure is described in the next section.

\section{The Correct Design Procedure Leads to an Underspecified Problem}

The first design step is to calculate the diameter of the first branch. To do this, the designer must assign the number of sectors, $z$, in which the disc is divided: This choice is obviously arbitrary, and it may depend on technological issues. Assuming $R e_{i n}=R e 0$ :

$$
d_{0}=\frac{4 m_{i n}}{z_{0} \pi \mu R e_{0}}
$$

Now, the designer must specify the total number of branchings, $z$. This choice is also arbitrary, but an obvious consideration is that the more branchings, the more outlet points there will be on the disc periphery, the more uniform the disc temperature will be and the most effective the cooling. In practice, this choice is limited by technological considerations about the minimum feasible diameter of the smallest branches (see below). Once $z b$ has been selected, the configuration of the disc is schematically represented in Figure 4 . 


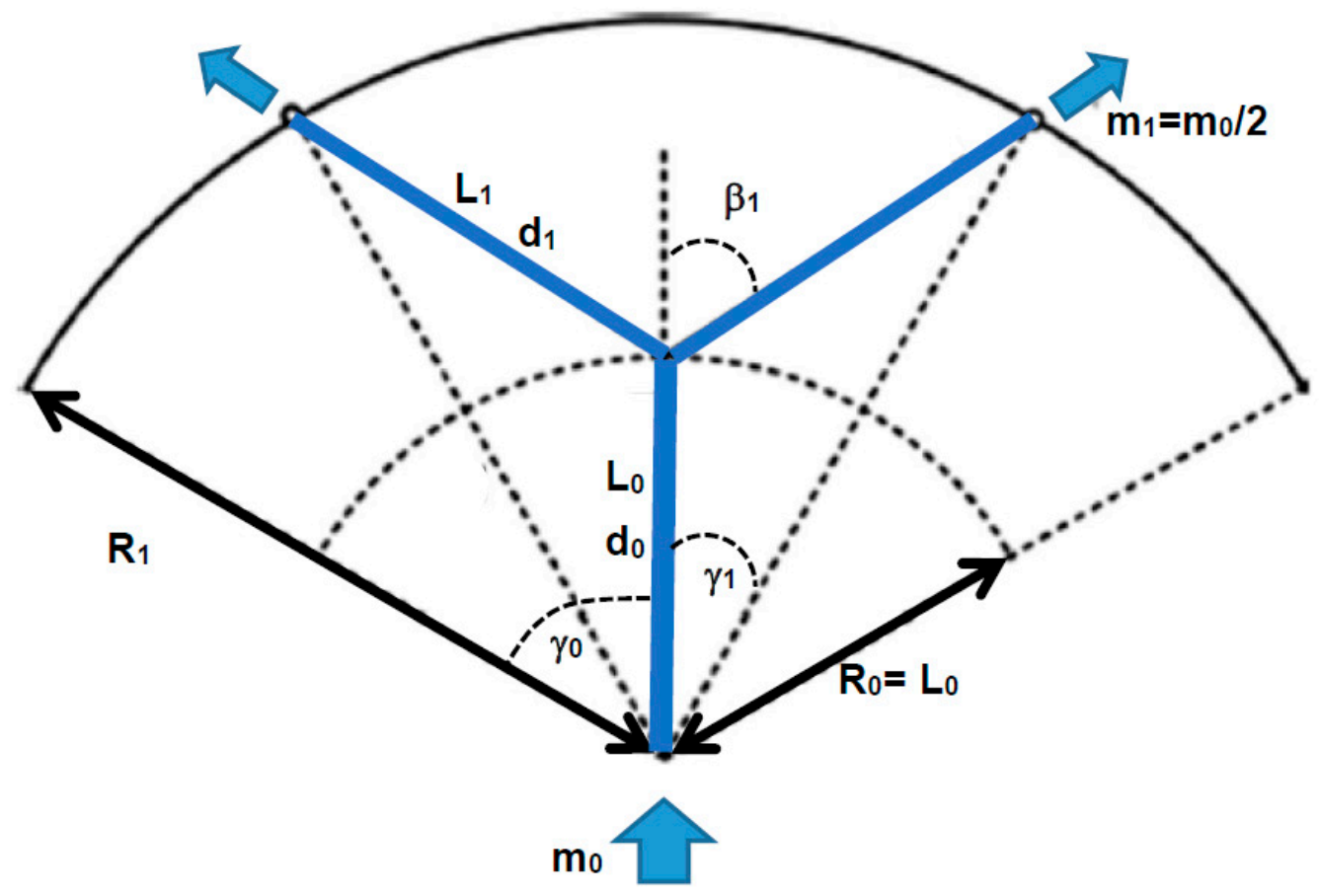

Figure 4. DSHE nomenclature (here, $\mathrm{z}_{0}=3, \gamma_{0}=60^{\circ}$ ).

For a given surface quality (that depends on material and technological considerations), the internal coefficient of heat transfer in each channel depends on the Nusselt number that, in turn, is a (non-linearly growing) function of the Reynolds number: For the generic $j$-th branch:

$$
N u_{j}=\frac{h_{j} d_{j}}{k}=f(R e, P r)
$$

Since it is obviously convenient to have as high a $N u_{j}$ as possible, a good design choice is to arrange the slenderness $d_{j} / L_{j}$ of the channels in such a way that the flow in all of the $j$-th branches is within the respective Graetz entry lengths (Figure 5): In other words, $L_{j}=\kappa_{g} d_{j}$, with $\kappa_{g}$ arbitrarily chosen in the shaded portion of the graph of Figure 5.

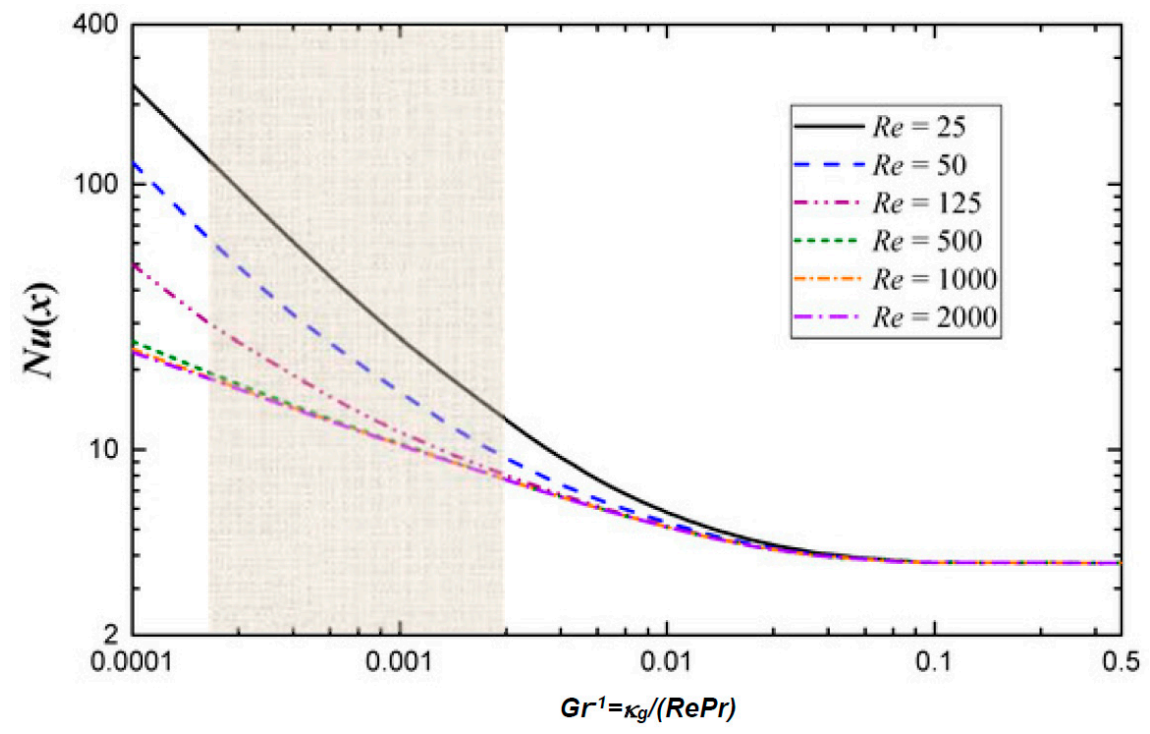

Figure 5. Nusselt number vs. Graetz number (adapted from [28]).

On the other hand, the friction losses are, under the posited assumptions, also a growing function of $R e$ : 


$$
\frac{\Delta p_{j}}{L_{j}}=f(R e)
$$

Therefore, the design choice poses the well-known dilemma of the HE designer: Higher pressure losses vs. higher heat transfer coefficient. Previous theoretical work on bifurcated structures $[29,30]$ suggests imposing $R e_{j}=R e_{0}$, which leads to:

$$
\delta_{j}=1 / 2
$$

Thus, the diameter of the internal channels halves at every new split: This is the reason for which $z_{b}$ has an upper bound, posed both by Equation (6) and by possible technological limitations on the attainable surface roughness. For a given Graetz ratio (for circular tubes, the Graetz entry length varies between 10 and 15 diameters $(\kappa=7 \div 15)$, depending on the boundary conditions on the tube wall and the flow structure. In most practical applications, a value $\kappa_{\mathrm{g}} \approx 8-10$ is satisfactory, see Section 5 below):

$$
L_{j}=\kappa_{g} d_{j}=\kappa_{g} d_{0} \delta^{j} \text { and } L_{j+1}={ }^{L_{j}} / 2
$$

with the geometric parameters thus defined, from simple trigonometric manipulations it follows that the radii of the $z b$ circumferences identified by each splitting level are given by a recursive formula:

$$
R_{z_{b}}=R ; \quad \mathrm{R}_{j-1}=\mathrm{R}_{j} \cos \left(\gamma_{j}\right)+\kappa_{g} d_{0} \delta^{j} \cos \left(\beta_{j}\right)
$$

A graphical representation for the structures defined by Equation (9) is provided in Figure 6. Equation (9) solves the problem because $R_{z_{b}}$ and $d_{0}$ are known (Equations (2) and (4)), but it contains $z_{b}$ unknowns, namely, the splitting angles $\beta_{j}$. To close the problem, $z_{b}-1$ auxiliary conditions must be specified.

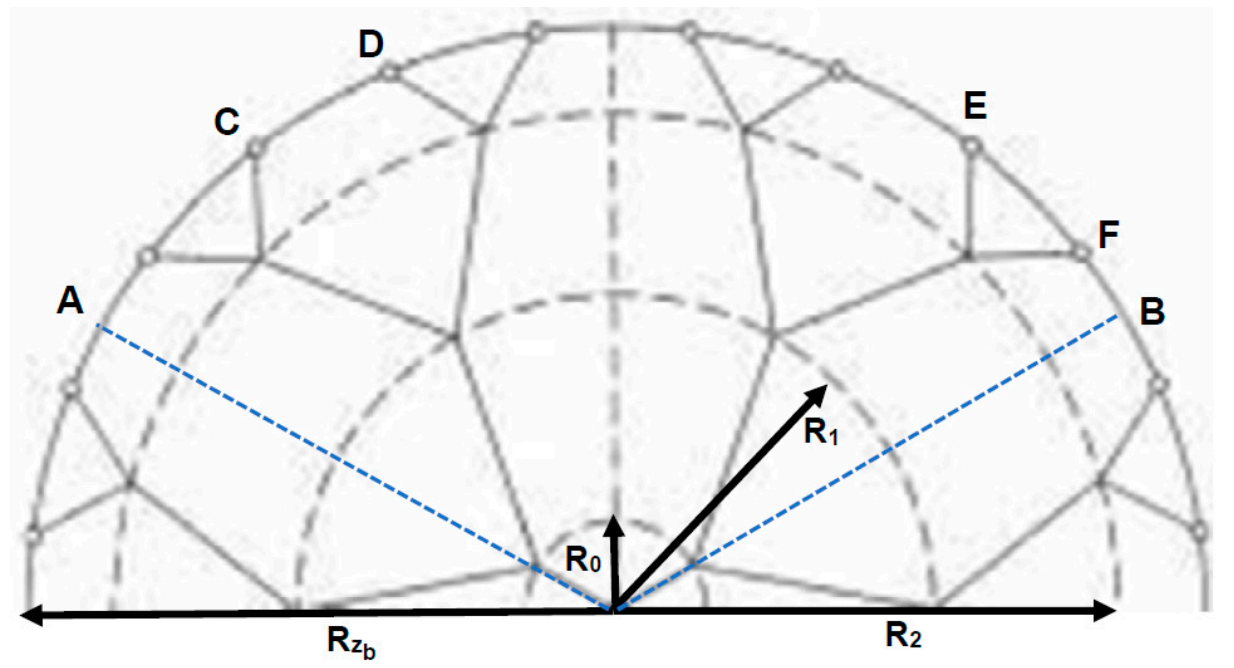

Figure 6. Identification of the radii of successive branchings (here, $\mathrm{z}_{0}=3$ ).

\section{The Proper Constraints}

Once the initial number of branches (level 0), $z_{0}$, and the total number of branchings $z_{b}$ are specified, a series of geometric constraints can be derived that completely defines the configuration by providing an equation for each of the $\beta_{j}$. The line of reasoning is as follows: The circular arc $\widehat{A B}$ at the external radius $R_{z_{b}}$ defined by $\gamma_{z_{\mathrm{b}}}$ will contain (Figure 6) $2^{z_{b}}$ terminal points, each one being the outlet of a single channel. For all of the terminal points on the circumference to be equispaced, the central angle $\gamma_{z_{b}}$ spanned by two adjacent terminal points $\langle C, D\rangle$ (with $\widehat{C D}=\widehat{E F}$ ) must be equal to $\frac{\pi}{z_{0} 2^{\left(z_{b}-1\right)}}$. Then, by simple trigonometric considerations, and making use of Equations (7) and (8): 


$$
\begin{gathered}
\cos \left(\beta_{1}\right)=\frac{\frac{R_{1}\left(1-\cos \left(\gamma_{1}\right)\right.}{\kappa_{g} d_{0}}-1}{\delta} \\
\cos \left(\beta_{2}\right)=\frac{R_{2} \cos \left(\gamma_{2}\right)-R_{1}}{\kappa_{g} d_{0} \delta^{2}} \\
\cdots \cdots \cdots \cdots \cdots \cdots \cdots \cdots \cdots \cdots \cdots \cdots \cdots \cdots \cdots \cdots \cdots \cdots \cdots \cdots \cdots \cdots \cdots \\
\cos \left(\beta_{j}\right)=\frac{R_{j+1} \cos \left(\gamma_{j+1}\right)-R_{j}}{\kappa_{g} d_{0} \delta^{j+1}} \\
\cos \left(\beta_{z_{b}}\right)=\frac{R_{z_{b}} \cos \left(\gamma_{z_{b}}\right)-R_{z_{b}-1}}{\kappa_{g} d_{0} \delta^{z_{b}+1}}
\end{gathered}
$$

The set (10) contains zb equations that together with the second Equation in (9) and Equation (2) make the problem position complete. Consider that the angles $\gamma_{0} \ldots \gamma_{j} \ldots \gamma_{\mathrm{z}_{\mathrm{b}}}$ are given by the recursive formula

$$
\gamma_{j}=\frac{\pi}{2^{(j-1)} z_{0}}
$$

\section{Examples of Application}

To demonstrate the usefulness of the above procedure, two samples of DSHE are critically examined in this section. Both are real applications, actually built in different years by different teams in one of the Thermal Sciences laboratories of the University Roma Sapienza. Independently from one other, both configurations were "optimized" according to two different criteria: Our goal here is to assess whether these configurations are indeed optimal, and in what sense.

\subsection{Comparison of Possible Dshe Configurations for an Oil Cooler}

In this application [31] the DSHE is used as an oil cooler: cold oil (iso viscosity class 46) enters

\begin{tabular}{|c|c|c|c|}
\hline Disc radius, $\mathrm{m}$ & 0.075 & Oil mass flow rate, $\mathrm{kg} / \mathrm{s}$ & $2.17 \times 10^{-3}$ \\
\hline Disc thickness, $\mathrm{m}$ & 0.015 & Oil density at inlet, $\mathrm{kg} / \mathrm{m}^{3}$ & 920 \\
\hline Disc conductivity $\mathrm{k}, \mathrm{W} /(\mathrm{mK})$ & 190 & Oil viscosity, $v_{\text {oil }, 323 \mathrm{~K}}, \mathrm{~m}^{2} / \mathrm{s}$ & $6.7 \times 10^{-5}$ \\
\hline Texternal AIR, $\mathrm{K}$ & 293 & Toil,in, $\mathrm{K}$ & 301 \\
\hline $\begin{array}{c}\text { External air viscosity vair, } \\
\mathrm{m}^{2} / \mathrm{s}\end{array}$ & $1.56 \times 10^{-5}$ & Toil,out, $_{\text {K }}$ & 349 \\
\hline
\end{tabular}
the disc from the axis (Figure 7) and is heated as it flows through the internal channels. The al-mn disc is subjected to a uniform heat flux on its bottom surface, the upper one being insulated. The case specifications are listed in Table 2.

Table 2. An Al/Mn alloy disc-shaped oil heater. 


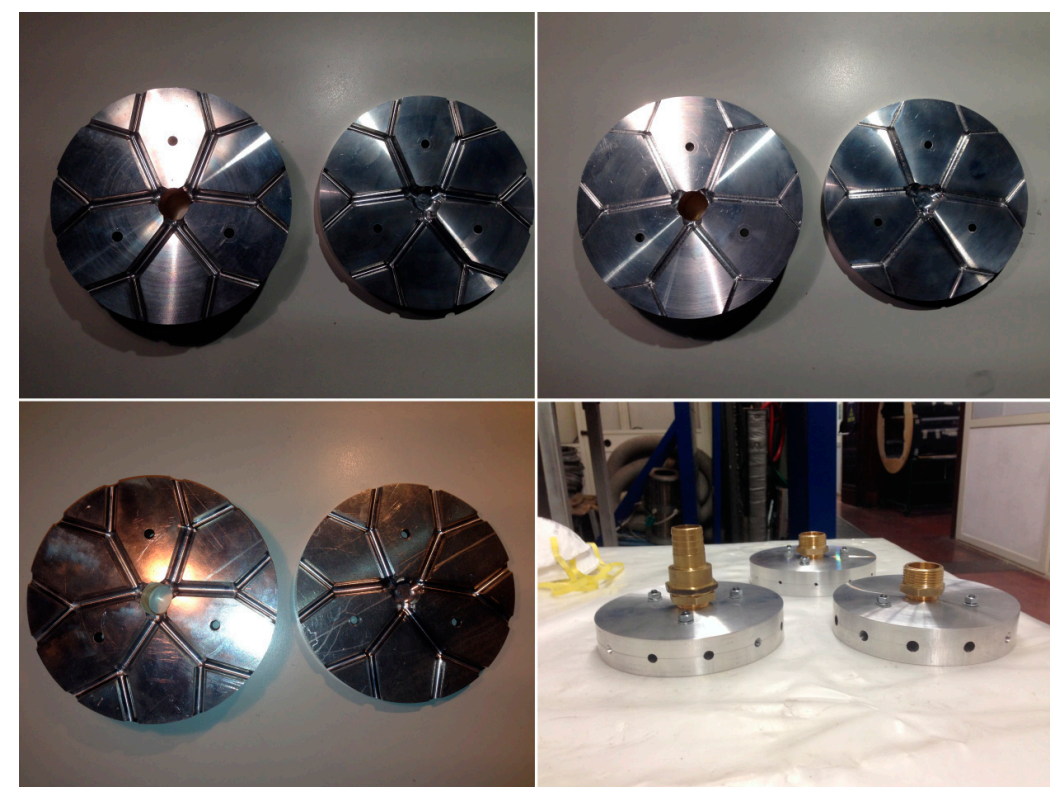

Figure 7. The DSHE described in [31].

The above device has been built and tested [31]. However, application of the procedure described in the previous sections demonstrates that:

(a) The 3 branches at level 0 are far too short, and therefore, the temperature of the portion of the disc within the radius $R_{0}$ (refer to Figure 6) is excessively high (the heat exchange area $z_{0} \pi d_{0} L_{0}$ is too small, even if the $N u_{0}$ is rather high);

(b) Since the branches at levels 1 and 2 do not respect the constant-Re prescription $(\delta=\sqrt{2} / 2$, i.e., constant velocity in successive branches), there is a disuniformity in the heat transfer (and therefore, in the disc bulk temperature) between $\mathrm{R}_{1}$ and $\mathrm{R}_{2}$;

(c) The $\kappa_{\mathrm{g}}$ is not the same in branches 1 and 2, and this adds up to the non-constant Re effect, increasing the disuniformity in the disc body.

\subsection{Selection of the DSHE Configuration for the Cooling of an Electronic Chip}

In this application [1] the DSHE is made of an Al-alloy and serves as the cooling unit of an electronic chip. It receives a constant heat flux $(14 \mathrm{~W})$ on its upper external surfaces, and it must be conservatively designed in such a way that its external temperature never exceeds $350 \mathrm{~K}$ when the disc lower external surface is insulated. The cooling air from a fan enters the disc from the axis (Figure 8 ) and flows through three sectors of equal amplitude $\left(\mathrm{z}_{0}=3\right)$, each shaped as a single split ( $\mathrm{zb}$ $=2$ ). Design specifications are listed in Table 3 .
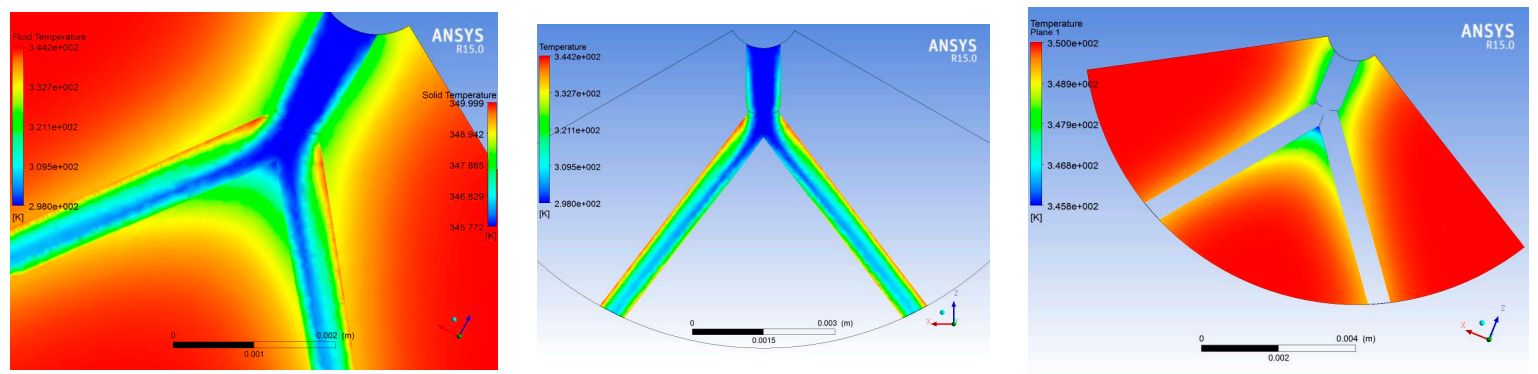

Figure 8. Ultra-micro DSHE: Results of a RANS CFD simulation (adapted from [5]). 
Table 3. An air-cooled ultra-micro DSHE.

\begin{tabular}{|c|c|c|c|}
\hline Disc radius, $\mathrm{m}$ & 0.015 & Air mass flow rate, $\mathrm{kg} / \mathrm{s}$ & $1.27 \times 10^{-4}$ \\
\hline Disc thickness, m & 0.0014 & Air density at inlet, $\mathrm{kg} / \mathrm{m}^{3}$ & 1.157 \\
\hline Disc conductivity $\mathrm{k}, \mathrm{W} /(\mathrm{mK})$ & 120 & Air viscosity $v_{\text {air }, 298,} \mathrm{~m}^{2} / \mathrm{s}$ & $1.56 \times 10^{-5}$ \\
\hline External h.t. coefficient $h, W /\left(\mathrm{m}^{2} \mathrm{~K}\right)$ & 30 & $\mathrm{~T}_{\text {air,in, }} \mathrm{K}$ & 298 \\
\hline Texternal air, $\mathrm{K}$ & 293 & $\mathrm{~T}_{\text {air,out, }} \mathrm{K}$ & 324 \\
\hline
\end{tabular}

The results of the CFD simulation show that:

(a) The level 0 branch is too short: the flow is not completely developed, and the Nu is high, but, as in the case examined in Section 5.1 above, the heat transfer is not optimal (the portion of disc within the circle $\mathrm{R}_{0}$ is too hot);

(b) The branches at level 1 are, on the contrary, too long, and the flow becomes fully developed towards the end of the channels, lowering the overall $\mathrm{Nu}$;

(c) In spite of the above shortcomings, the temperature of the disc is approximately constant, validating the assumption made in Section 2 as to the external convection loss.

\section{Conclusions}

A simple procedure for the design of a disc-shaped heat exchanger with internal bifurcated channels is presented and discussed. The goal of this exercise is to show that, while the study of the onset and operation of bifurcated structures in nature requires accurate considerations of optimal energy (exergy) use ratios, when it comes to engineered devices, it is not always necessary to "mimic nature", as many authors (including the present one!) have repeatedly stated. Diligent engineering considerations, and a small number of prime principles in fluid and thermodynamics suffice to concoct a sufficiently accurate preliminary design that is as "optimal" as needed. This happens by no chance: In the case of heat exchanger design, examined in this work, the specifications are quite stringent, and if additional symmetries are imposed by the selection of the device shape, the "design degrees of freedom" drop to zero. This does not mean that a design activity is devoid of creativity: In fact, as shown here, some of such "creativity" is naturally embedded in engineering choices that an expert designer deems "natural", and that are instead the result of an engineering culture that is perceived as implicit in the designer's reasoning.

\section{References}

1. Boichot, R.; Fan, Y. A genetic algorithm for topology optimization of area-to-point heat conduction problem. Int. J. Thermal Sci. 2016, 108, 209-217.

2. Feng, H.-J.; Chen, L.-G.; Xie, Z.-H.; Sun, F.R. Constructal entropy generation rate minimization for asymmetric vascular networks in a disc-shaped body. Int. J. Heat Mass Transf. 2015, 91, 1010-1017.

3. Hess, W.R. Eine mechanisch bedingte Gesetzmäßigkeit im Bau des Blutgefäßsystems, (A mechanically-based general rule for the construction of the blood circulatory system). Arch. Entwicklungsmech. Org. 1903, 16, 632-641.

4. Miguel, A.F. A study of entropy generation in tree-shaped flow structures. Int. J. Heat Mass Transf. 2016, 92, 349-359.

5. Murray, C.D. The physiological principle of minimum work applied to the angle of branching of arteries. J. General Physiol. 1926, 9, 835-841.

6. Murray, C.D. The physiological principle of minimum work-I: The vascular system and the cost of blood volume. Proc. Natl. Acad. Sci. USA 1926, 12, 207-214.

7. Hess, W.R. Über die periphere Regulierung der Blutzirkulation, (On the peripheral regulatory actions of blood circulation), Pflügers Archiv für die gesamte Physiologie des Menschen und der Tiere, Aug 1917, v.168, n.9, 439-490.

8. Murray, C.D. The physiological principle of minimum work-II: Oxygen exchange in capillaries. Proc. Natl. Acad. Sci. USA 1926, 12, 299-304. 
9. Robbe, M.; Sciubba, E.; Bejan, A.; Lorente, S. Numerical analysis of a tree-shaped cooling structure for a 2-D slab: A validation of a "constructally optimal" configuration. In Proceedings of the ESDA-ASME 8th Conference, Torino, Italy, 4-7 July 2006.

10. Sciubba, E. A Critical Reassessment of the Hess-Murray Law. Entropy 2016, 18, 283-300.

11. Wechsatol, W.; Lorente, S.; Bejan, A. Dendritic convection on a disc. Int. J. Heat Mass Transf. 2003, 46, 43814391.

12. Sciubba, E. Entropy Generation Minimization as a Design Tool. Part 1: Analysis of different configurations of branched and non-branched laminar isothermal flow through a circular pipe. Int. J. Thermodyn. 2011, 14, 11-20.

13. Bejan, A. Constructal-theory network of conducting paths for cooling a heat generating volume. Int. J. Heat Mass Transf. 1997, 40, 799-816.

14. Capata, R.; Beyene, A. Experimental evaluation of three different configurations of constructal disc-shaped heat exchangers. Int. J. Heat Mass Transf. 2017, 115, 92-101.

15. da Silva, A.K.; Bejan, A. Dendritic counterflow heat exchanger experiments. Int. J. Thermal Sci. 2006, 45, 860-869.

16. Su, L.-B.; Duan, Z.-P.; He, B.-S.; Ma, H.; Xu, Z. Thermally Developing Flow and Heat Transfer in Elliptical Minichannels with Constant Wall Temperature. Micromachines 2019, 10, 713-728.

17. Wechsatol, W.; Lorente, S.; Bejan, A. Optimal tree-shaped networks for fluid flow in a disc-shaped body. Int. J. Heat Mass Transf. 2002, 45, 4911-4924.

18. Bejan, A.; Alalaimi, M.; Sabau, A.S.; Lorente, S. Entrance-length dendritic plate heat exchangers. Int. J. Heat Mass Transf. 2017, 114, 1350-1356.

19. Bejan, A.; Sciubba, E. The optimal spacing of parallel plates cooled by forced convection. Int. J. Heat Mass Transf. 1992, 35, 3259-3264.

20. Bejan, A.; Lorente, S. Constructal theory of energy-system and environment flow configurations. Int. J. Exergy 2005, 2, 335-347.

21. Luo, L. (Ed.) Heat and Mass Transfer Intensification and Shape Optimization: A Multi-scale Approach; Springer: London, UK, 2013.

22. Mereu, S.; Sciubba, E.; Bejan, A. The optimal cooling of a stack of heat generating boards with fixed pressure drop, flowrate or pumping power. Int. J. Heat Mass Transf. 1993, 6, 3677-3686.

23. Qureshi, M.U.; Vaughan, G.D.A.; Sainsbury, C.; Johnson, M.; Peskin, C.S.; Olufsen, M.S.; Hill, N.A. Numerical simulation of blood flow and pressure drop in the pulmonary arterial and venous circulation. Biomech. Model. Mechanobiol. 2014, 13, 1137-1154.

24. Robbe, M.; Sciubba, E. Derivation of the optimal internal cooling geometry of a prismatic slab: Comparison of constructal and non-constructal geometries. Energy 2009, 34, 2167-2174.

25. West, G.B.; Brown, J.H. The origin of allometric scaling laws in biology from genomes to ecosystems: Towards a quantitative unifying theory of biological structure and organization. J. Exp. Biol. 2005, 208, 1575-1592.

26. Zamir, M.; Wrigley, S.M.; Langille, B.L. Arterial bifurcations in the cardiovascular system of a rat. J. General Phys. 1983, 81, 325-335.

27. Errera, M.R.; Bejan, A. Deterministic tree networks for river drainage basins. Fractals 1998, 6, $245-261$.

28. Sherman, T.F. On Connecting Large Vessels to Small: The Meaning of Murray's Law. J. General. Physiol. 1981, 78, 431-453.

29. Roy, A.G.; Woldenberg, M.J. A generalization of the optimal models of arterial branching. Bull. Math. Biol. 1982, 44, 349-360.

30. Sciubba, E. Entropy Generation Minima in Different Configurations of the Branching of a Fluid-Carrying Pipe in Laminar Isothermal Flow. Entropy 2010, 12, 1855-1866. 
31. Cancellario, A. Waste Heat Recovery System at Nanoscales: Process Simulation, Optimization, Design of Heat Exchanger. Master's Thesis, U. Roma Sapienza, Rome, Italy. 2017.

Publisher's Note: MDPI stays neutral with regard to jurisdictional claims in published maps and institutional affiliations.

(c) 2020 by the authors. Licensee MDPI, Basel, Switzerland. This article is an open access article distributed under the terms and conditions of the Creative Commons Attribution (CC BY) license (http://creativecommons.org/licenses/by/4.0/). 